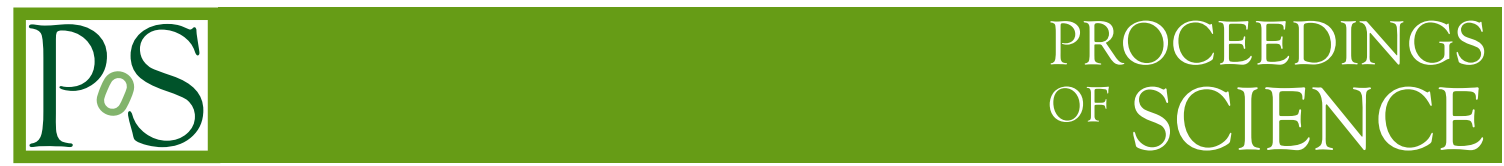

\title{
Mixing and CP-violation studies in charm decays at $\mathrm{LHCb}$
}

\section{Matthew CHARLES*}

On behalf of the LHCb collaboration

The University of Oxford

E-mail: m.charles1ephysics.ox.ac.uk

Studies of charm physics with the $2010 \mathrm{LHCb}$ data sample are presented. Time-integrated searches for CP violation in $D^{+} \rightarrow K^{-} K^{+} \pi^{+}$and $D^{0} \rightarrow K^{-} K^{+}, \pi^{-} \pi^{+}$are discussed.

The 2011 Europhysics Conference on High Energy Physics, EPS-HEP 2011,

July 21-27, 2011

Grenoble, Rhône-Alpes, France

* Speaker. 

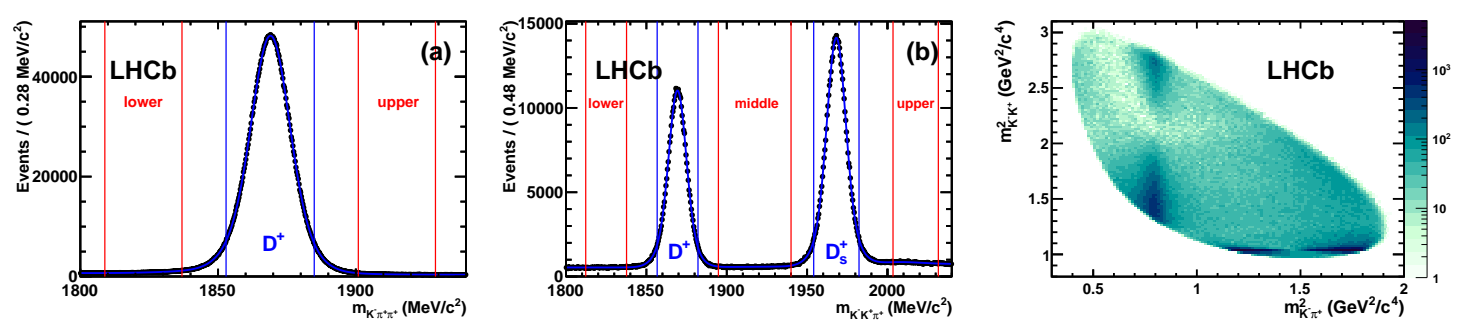

Figure 1: Mass spectra and Dalitz plot. The mass spectra after selection for (a) $K^{-} \pi^{+} \pi^{+}$and (b) $K^{-} K^{+} \pi^{+}$ are shown, with the signal and sideband mass windows indicated. For those candidates in the $D^{+} \rightarrow$ $K^{-} K^{+} \pi^{+}$signal window, the Dalitz plot is shown on the right.

\section{Introduction}

The charm sector is a promising place to probe for new physics effects. Mixing is now wellestablished [1] at a level which is consistent with but at the upper end of Standard Model (SM) expectations [2]. Three types of $C P$ violation (CPV) are possible: in the decay amplitudes, in the mixing between $D^{0}$ and $\bar{D}^{0}$, and in the interference between mixing and decay. The first is referred to as direct CPV, and the second and third as indirect CPV. Only direct CPV is possible in $D^{+}$decays, due to the absence of mixing. In the SM indirect $C P$ violation is expected to be small and direct $C P$ violation in singly-Cabibbo-suppressed modes such as those discussed below is naively expected to be $\mathscr{O}\left(10^{-3}\right)$ or less [3], though larger values cannot be excluded from first principles [4]. In the presence of new physics the rate of $C P$ violation could plausibly be enhanced to $\mathscr{O}\left(10^{-2}\right)$. At the time of the conference no evidence for $\mathrm{CPV}$ in charm had yet been found, though first indications have since emerged in the 2011 LHCb data [5].

\section{Search for CPV in $D^{+} \rightarrow K^{-} K^{+} \pi^{+}$}

Direct $C P$ violation arises when two different amplitudes with non-zero relative weak and strong phases contribute to decays to the same final state. In two-body decays this must imply contributions from different Feynmann diagrams, such as from tree and penguin processes. In multi-body decays the same mechanism exists, but in addition a rich variety of intermediate resonant states can contribute to the decay, each naturally producing a different strong phase with well-defined variation across the Dalitz plane. Thus, the interference between these amplitudes can give rise to observable asymmetries which change across the Dalitz plane.

We search for such asymmetries at LHCb [6] by comparing the Dalitz plot distributions of $D^{+} \rightarrow K^{-} K^{+} \pi^{+}$and its conjugate process $D^{-} \rightarrow K^{+} K^{-} \pi^{-}$(Fig. 1), applying a model-independent technique of comparing the binned, normalized distributions. Normalizing the two Dalitz plots to the same total number of events cancels any production asymmetry and suppresses many systematic effects that are mainly expressed as an overall efficiency asymmetry. The statistical technique used to test for consistency between the $D^{+}$and $D^{-}$Dalitz plots, and to localize the asymmetry if one is found, is based on the Miranda approach (see Ref. [7] and also Ref. [8]). A variety of different binnings are used in order to test for different manifestations of $C P$ violation. 


\begin{tabular}{cccc}
\hline \hline Binning & Bins & $\chi^{2} /$ ndf & $p$-value (\%) \\
\hline Adaptive I & 25 & $32.0 / 24$ & 12.7 \\
Adaptive II & 106 & $123.4 / 105$ & 10.6 \\
Uniform I & 199 & $191.3 / 198$ & 82.1 \\
Uniform II & 530 & $519.5 / 529$ & 60.5 \\
\hline \hline
\end{tabular}

Table 1: $\chi^{2} /$ ndf and $p$-values for consistency with no CPV for the $D^{+} \rightarrow K^{-} K^{+} \pi^{+}$decay mode with four different binnings.

Control modes are analysed to validate the method. The main tool is the Cabibbo-favoured $D_{s}^{+} \rightarrow K^{-} K^{+} \pi^{+}$control mode, which has the same final state as the signal as well as similar kinematics and Dalitz plot structure. As expected, no evidence of any asymmetry is found in this mode (e.g. p-value of $34 \%$ for 25 -bin adaptive binning), nor in the sidebands around the $D^{+}$mass window. In addition, the analysis is repeated for the Cabibbo-favoured $D^{+} \rightarrow K^{-} \pi^{+} \pi^{+}$mode. This is more sensitive to systematic effects, since (a) the yield is ten times larger than that of the signal mode, and (b) the kaon imbalance can induce momentum-dependent detector efficiency asymmetries which would not be present in the signal mode. Nonetheless, only weak indications of asymmetries are seen (e.g. p-value of $12 \%$ for 25 -bin adaptive binning). Thus, systematic effects in the more robust $D^{+} \rightarrow K^{-} K^{+} \pi^{+}$signal mode are negligible. The final, unblinded results are shown in Table 1: no evidence of $C P$ violation is found in the 2010 data. For further details, see Ref. $[9,5]$.

\section{Search for CPV in $D^{0} \rightarrow K^{-} K^{+}, \pi^{-} \pi^{+}$}

As discussed in Section 1, both direct and indirect CPV can contributed to the time-integrated $C P$ asymmetry in these singly Cabibbo suppressed decays to $C P$-even final states. The indirect $C P$ asymmetry is universal to a very good approximation [10], although the measured value is affected by the $D^{0}$ decay time acceptance of the experiment [11]. However, the direct $C P$ asymmetry in general varies between final states, and in the limit of U-spin symmetry is equal and opposite between $K^{-} K^{+}$and $\pi^{-} \pi^{+}$[3]. Thus, the difference in time-integrated asymmetry between the two final states, $\triangle A_{C P}$, is sensitive to direct CPV but has limited sensitivity to indirect CPV:

$$
\Delta A_{C P}=a_{C P}^{\mathrm{dir}}\left(K^{-} K^{+}\right)-a_{C P}^{\mathrm{dir}}\left(\pi^{-} \pi^{+}\right)+\frac{\Delta\langle t\rangle}{\tau} a_{C P}^{\mathrm{ind}},
$$

where $\frac{\Delta\langle t\rangle}{\tau}=0.10 \pm 0.01$ is the difference in normalized time acceptance for the two final states at LHCb, $a_{C P}^{\mathrm{dir}}(f)$ is the direct $C P$ asymmetry for final state $f$, and $a_{C P}^{\text {ind }}$ is the indirect $C P$ asymmetry.

The observable $\Delta A_{C P}$ also has the advantage of being highly robust against systematic effects. The measured (raw) asymmetry between $D^{0} \rightarrow f$ and $\bar{D}^{0} \rightarrow \bar{f}$, where the initial flavour of the $D$ is established with a $D^{*+} \rightarrow D^{0} \pi_{\mathrm{s}}$ tag, can be written at first order as:

$$
A_{\mathrm{RAW}}(f) \approx A_{C P}(f)+A_{\mathrm{D}}(f)+A_{\mathrm{D}}\left(\pi_{\mathrm{s}}\right)+A_{\mathrm{P}}\left(D^{*+}\right)
$$

where $A_{C P}, A_{\mathrm{D}}$, and $A_{\mathrm{P}}$ are the relevant physics, detector efficiency, and production asymmetries, respectively. Within a local kinematic region, $A_{\mathrm{D}}\left(\pi_{\mathrm{s}}\right)$ and $A_{\mathrm{P}}\left(D^{*+}\right)$ are independent of the $D^{0}$ de- 
cay mode and thus cancel in the difference $\Delta A_{C P}$. Further, $A_{\mathrm{D}}\left(K^{-} K^{+}\right)$and $A_{\mathrm{D}}\left(\pi^{-} \pi^{+}\right)$are zero by construction, since the final state is spinless and self-conjugate. Thus, all detector and production effects cancel in $\Delta A_{C P}$ at first order. To ensure good behaviour at second order, the data are divided into 12 disjoint kinematic bins, as well as being partitioned according to trigger conditions and magnetic field polarity. Taking the weighted average of the individual measurements, we obtain $\Delta A_{C P}=(-0.28 \pm 0.70 \pm 0.25) \%$, where the first uncertainty is statistical and the second is systematic (taking into account modeling of the lineshapes [0.06\%], the $D^{0}$ mass window [0.20\%], multiple candidates [0.13\%], and the kinematic binning [0.01\%]). For further details, see Ref. [12].

\section{Conclusions and prospects}

LHCb's charm physics programme is off to a strong start. Several proof-of-concept measurements have been made on the 2010 data sample of $38 \mathrm{pb}^{-1}$, and the first results on the much larger 2011 and 2012 data sets are now forthcoming.

\section{References}

[1] Heavy Flavor Averaging Group, D. Asner et al., Averages of b-hadron, c-hadron, and $\tau$-lepton Properties, arXiv:1010.1589.

[2] A. F. Falk, Y. Grossman, Z. Ligeti, Y. Nir and A. A. Petrov, The $D^{0}-\bar{D}^{0}$ mass difference from a dispersion relation, Phys. Rev. D 69 (2004) 114021, [hep-ph/0402204].

[3] See e.g. S. Bianco, F. L. Fabbri, D. Benson and I. Bigi, A Cicerone for the physics of charm, Riv. Nuovo Cim. 26N7 (2003) 1 [hep-ex/0309021]; M. Bobrowski, A. Lenz, J. Riedl and J. Rohrwild, How large can the SM contribution to CP violation in $D^{0}-\bar{D}^{0}$ mixing be?, JHEP 1003 (2010) 009 [arXiv:1002.4794]; Y. Grossman, A. L. Kagan and Y. Nir, New physics and CP violation in singly Cabibbo suppressed D decays, Phys. Rev. D 75 (2007) 036008 [hep-ph/0609178].

[4] J. Brod, A. L. Kagan and J. Zupan, On the size of direct CP violation in singly Cabibbo-suppressed D decays, arXiv:1111.5000.

[5] LHCb Collaboration, R. Aaij et al., Evidence for CP violation in time-integrated $D^{0} \rightarrow h^{-} h^{+}$decay rates, arXiv:1112.0938 (submitted to Phys. Rev. Lett.).

[6] LHCb Collaboration, A. A. Alves, Jr. et al., The LHCb Detector at the LHC, JINST 3 (2008) S08005.

[7] I. Bediaga, I. I. Bigi, A. Gomes, G. Guerrer, J. Miranda and A. C. d. Reis, On a CP anisotropy measurement in the Dalitz plot, Phys. Rev. D 80 (2009) 096006 [arXiv:0905.4233].

[8] BABAR Collaboration, B. Aubert et al., Search for CP Violation in Neutral D Meson Cabibbo-suppressed Three-body Decays, Phys. Rev. D 78 (2008) 051102 [arXiv:0802.4035].

[9] LHCb Collaboration, R. Aaij et al., Search for CP violation in $D^{+} \rightarrow K^{-} K^{+} \pi^{+}$decays, arXiv:1110.3970 (accepted by Phys. Rev. D).

[10] A. L. Kagan and M. D. Sokoloff, On Indirect CP Violation and Implications for $D^{0}-\bar{D}^{0}$ and $B_{s}^{0}-\bar{B}_{s}^{0}$ mixing, Phys. Rev. D 80 (2009) 076008 [arXiv:0907.3917].

[11] CDF Collaboration, T. Aaltonen et al., Measurement of CP-violating asymmetries in $D^{0} \rightarrow \pi^{+} \pi^{-}$and $D^{0} \rightarrow K^{+} K^{-}$decays at $C D F$, arXiv:1111.5023.

[12] LHCb Collaboration, A search for time-integrated CP violation in $D^{0} \rightarrow h^{+} h^{-}$decays and a measurement of the $D^{0}$ production asymmetry, LHCb-CONF-2011-023. 